\title{
Construction of a Simple Rectum Model Using Image Guidance in Prostate Patients Treated with 3D Conformal Radiotherapy
}

\author{
Marco D’Andrea1*, Maria Daniela Falco², Dahlia Fedele², Elisabetta Ponti², Barbara Tolu², \\ Daniela Di Cristino², Rosaria Barbarino², Luana Di Murro², Grazia Tortorelli2, \\ Andrea Duggento ${ }^{3}$, Paolo Bagalà ${ }^{2,3}$, Maria Guerrisi ${ }^{3}$, Riccardo Santoni2 \\ ${ }^{1}$ Laboratory of Medical Physics and Expert Systems, National Cancer Institute Regina Elena, \\ Rome, Italy \\ ${ }^{2}$ Department of Diagnostic Imaging, Molecular Imaging, Interventional Radiology and Radiotherapy, \\ Tor Vergata University General Hospital, Rome, Italy \\ ${ }^{3}$ Department of Biomedicine and Prevention, Medical Physics Section, Tor Vergata University, \\ Rome, Italy \\ Email: ${ }^{*}$ dandrea@ifo.it
}

Received 15 August 2014; revised 10 September 2014; accepted 5 October 2014

Copyright (C) 2014 by authors and Scientific Research Publishing Inc.

This work is licensed under the Creative Commons Attribution International License (CC BY). http://creativecommons.org/licenses/by/4.0/

(c) $\underset{\mathrm{EY}}{\mathrm{BP}}$ Open Access

\section{Abstract}

Purpose: To evaluate the performance of a rectum model in predicting late rectal toxicity of prostate patients undergoing 3D conformal radiation therapy while following a dietary protocol combined with image guidance. Methods: A linear accelerator equipped with a Cone Beam Computed Tomography (CBCT) system was used to treat 20 patients who were following a dietary protocol. The set-up was verified by co-registering CBCT scans with the planning CT scan (pCT). A mean dose volume histogram $(<D V H>$ ) as the arithmetical mean of the rectum DVHs from each CBCT scan was obtained. A suitably defined 3D rectum model (Average Rectum, AR) was defined and its $\mathrm{DVH}\left(\mathrm{DVH}_{\mathrm{AR}}\right)$ was calculated. DVHs were also evaluated for the first five CBCT scans using both methods ( $<\mathrm{DVH}_{5}>$ and $\mathrm{DVH}_{\mathrm{AR}}$ ). The Lyman-Kutcher-Burman NTCP model with QUANTEC parameters was used to compare the calculated DVHs. The QUANTEC dose values were used to describe the time behaviour of the relative volumes using the Gamma Distribution for the frequency of the relative rectum volumes at each QUANTEC dose value. Results: No statistically significant differences between $\mathrm{NTCP}_{\mathrm{AR} 5}$ and $\mathrm{NTCP}_{\mathrm{AR}}$ and between $\mathrm{NTCP}_{<\mathrm{DVH} 5>}$ and $\mathrm{NTCP}<\mathrm{DVH}>$ were found. The best agreement with the observed toxicity rate $(0 \%)$ was obtained form $\mathrm{DVH}_{\mathrm{AR}}$. The Gamma Distributions of the rectum volumes at the QUANTEC dose levels were found to be highly variable among the patients. Conclusions: Both dietary protocol and image guidance were found effective in limiting late rectal toxicity. AR was a better predictor for late rectal toxicity and better described the ${ }^{*}$ Corresponding author.

How to cite this paper: D'Andrea, M., Falco, M.D., Fedele, D., Ponti, E., Tolu, B., Di Cristino, D., Barbarino, R., Di Murro, L., Tortorelli, G., Duggento, A., Bagalà, P., Guerrisi, M. and Santoni, R. (2014) Construction of a Simple Rectum Model Using Image Guidance in Prostate Patients Treated with 3D Conformal Radiotherapy. Journal of Cancer Therapy, 5, $1039-1048$. 
rectum volume during the treatment course. Finally, from the Gamma distributions, and from our toxicity data, we can suggest $V_{75}$ as the best predictor of late rectal toxicity.

\title{
Keywords
}

\author{
Organ Motion, Prostate Cancer, Rectal Toxicity, Radiotherapy
}

\section{Introduction}

Prostate carcinoma is the most common malignant and potentially curable tumour in males in western country and the most frequent cause of cancer death [1] [2]. Conformal radiotherapy techniques, such as Three-Dimensional Radiation Therapy (3D-CRT) and Intensity-Modulated Radiation Therapy (IMRT), allow dose escalation to the prostate and reduced side effects [3]-[5]. Rectal toxicity represents the most important side effect of prostate irradiation and impacts significantly on quality of life [6]-[9]. The analysis of the dose-volume histograms (DVH) of the rectum has been performed and some constraints have been proposed [10]-[17]. Author correlated the investigation constraints with late rectal bleeding in order to keep the risk of developing Grade 2 or worse toxicity as low as possible using different definitions for the rectum [18]-[20]. Most of these investigations that aimed at finding reliable constraints were built on 3D-CRT toxicity data and did not take into account information/guidance from on-board imaging systems, the latter being a technology not available at the time of collection of most data. In fact, internal motion between fractions can significantly influence dose accumulation over a course of treatment [21]-[24]. In this respect, a standardized IGRT protocol associated with a dietary regimen aimed at decreasing the volume of faeces and gas in the rectum can limit the inter- and intra-fraction prostate motion [25]. The surrounding normal tissues can be spared by reducing margins thus offering further opportunity for dose escalation [3] [4].

In a previous analysis [26], we have obtained Cone Beam Computed Tomography (CBCT) scans in six patients and assumed that those performed during the first week of treatment were representative of the whole treatment course. Both the arithmetical mean of the dose-volume histograms and the DVH of a geometrical model of the Average Rectum (AR) were in accordance within $\pm 5 \%$ and usually lower than the DVHs of the planning CT (pCT) scans, thanks to the dietary regimen. The aim of the present evaluation is to extend the same analysis to 20 patients in order to corroborate our preliminary results. To compare the DVHs arising from the different ways of handling the rectum contours we have used the Lyman Kutcher Burmann Normal Tissue Complication Probability (NTCP-LKB) model [27] with the parameter values proposed by the QUANTEC (Quantitative Analysis of Normal Tissue Effects in the Clinic) [16] group. Finally, to obtain indications on how the daily filling of the rectum could impact on toxicity, we have also studied the time distributions of the CBCT scans and compared the cumulative DVH points at the dose values taken from the QUANTEC group.

\section{Materials and Methods}

A retrospective analysis on twenty clinical Stage I or II prostate cancer patients referred to our Radiation Oncology Unit between April 2008 and February 2010 was performed. The patients were instructed to follow a dietary regimen in order to reduce intestinal gas and to ensure an empty rectum both at the planning CT scan and at each treatment session. The diet was prescribed in combination with a daily mild laxative (1000 mg magnesium oxide) to be taken every night before going to bed [26]. Patient characteristics are summarized in Table 1.

Patients were treated in the supine position using $18 \mathrm{MV}$ photons produced by an Elekta Synergy ${ }^{\circledR} \mathrm{S}$ linear accelerator (Elekta, Crawley, UK) with an integrated 3D CBCT verification system. All patients received a dose of 76 Gy in 38 fractions (66 Gy were given to the prostate plus SVs (CTV1) and 10 Gy to the prostate only (CTV2)) using a 6-field 3D conformal technique. Treatment plans were developed using Pinnacle $8.0 \mathrm{~m}^{\circledR}$ (Philips Medical Systems, Cleveland, $\mathrm{OH}$ ). Our treatment dose-volume hard constraint for the rectum was that no more than $25 \%$ of its volume had to receive a dose of $70 \mathrm{~Gy}\left(\mathrm{~V}_{70} \leq 25 \%\right)$. As additional soft constraints no more than $60 \%$ of the rectal volume had to receive $40 \mathrm{~Gy}\left(\mathrm{~V}_{40} \leq 60 \%\right)$ and no more than $50 \%$ of the rectal volume had to receive 50 Gy $\left(\mathrm{V}_{50} \leq 50 \%\right)$.

All patients were scored according to the Radiation Therapy Oncology Group scoring criteria (RTOG/ 
Table 1. Patients' characteristics.

\begin{tabular}{ccc}
\hline Variables & & Patients \\
\hline Age & Median & 70.5 \\
Clinical state T & Range & $59-83$ \\
(AJCC 2002) & T1c-T2c & 19 \\
& T3a-T3b & 1 \\
PSA (ng/ml) & & 7.36 \\
& Median & $3.8-71.8$ \\
Gleason score & Range & 13 \\
& & 3 \\
Hormonal therapy LH-RH releasing & $2-6$ & 3 \\
Diabetes mellitus & 7 & 1 \\
Hemorrhoids & Not reported & 7 \\
Hypertension & Time 6 - 24 months in association to RT and in adjuvant & 5 \\
Inflammatory bowel disease & & 4 \\
\hline
\end{tabular}

EORTC), both for acute and late toxicity (Table 2).

Patients were examined weekly during the course of treatment, 8 weeks after treatment, every 4 months for the first 2 years and every 6 months afterwards. At each follow-up visit, physical examination including a digital rectal exam was performed and a questionnaire was used to collect information about urinary, anorectal morbidity and sexual function. The average follow-up was 35.3 months (range 12 - 58).

The set-up verification procedure, acquisition protocol and off line organ motion analysis have been extensively discussed in a previous report [26]. Briefly, CBCT scans were used in a routine online bone-anatomybased setup protocol. CBCT images were transferred to Pinnacle for off-line organ motion analysis [28]. The rectum outlines were used to obtain a mean DVH for each patient $(<\mathrm{DVH}\rangle)$ by averaging the DVHs of the rectum as they appeared in every CBCT scan. A geometrical model of the rectum, representative of the whole treatment, referred to as "Average Rectum" (AR), was obtained from the contours delineated on the CBCT scans and was used to calculate the corresponding $\mathrm{DVH}\left(\mathrm{DVH}_{\mathrm{AR}}\right)$. In addition both averaging methods were restricted to the first five CBCT scans only, representing the first week of treatment, and the corresponding DVHs were calculated $\left(<\mathrm{DVH}_{5}>\right.$ and $\mathrm{DVH}_{\mathrm{AR} 5}$, respectively).

To assess the influence of the dietary protocol on our results and to investigate the possibility of making reliable predictions on rectum position using data collected in the first week only we have compared $\mathrm{DVH}_{\mathrm{pCT}}$, $<\mathrm{DVH}_{5}>,<\mathrm{DVH}>, \mathrm{DVH}_{\mathrm{AR} 5}$ and $\mathrm{DVH}_{\mathrm{AR}}$, using the NTCP-LKB model to reduce the above mentioned DVHs into numbers. The following QUANTEC-recommended parameters for late effects [16] (95\% confidence intervals) were used in the calculation of NTCP-LKB (NTCP-QLKB): $\mathrm{n}=0.09 ; \mathrm{m}=0.13$ and $\mathrm{TD}_{50}=76.9 \mathrm{~Gy}$.

Pearson's correlation coefficients were calculated to measure how $\mathrm{NTCP}_{\mathrm{pCT}}, \mathrm{NTCP}_{<\mathrm{DVH} 5}, \mathrm{NTCP}_{<\mathrm{DVH}}$, $\mathrm{NTCP}_{\mathrm{AR} 5}$ and $\mathrm{NTCP}_{\mathrm{AR}}$, which are the NTCP values calculated from $\mathrm{DVH}_{\mathrm{pCT}},<\mathrm{DVH}_{5}>,<\mathrm{DVH}>, \mathrm{DVH}_{\mathrm{AR} 5}$ and $\mathrm{DVH}_{\mathrm{AR}}$ respectively, were pairwise related. To estimate the differences between medians of the NTCP distributions, i.e. to assess whether samples from $\mathrm{NTCP}_{\mathrm{pCT}}$, $\mathrm{NTCP}_{<\mathrm{DVH} 5>}, \mathrm{NTCP}_{<\mathrm{DVH}>} \mathrm{NTCP}_{\mathrm{AR} 5}$ and $\mathrm{NTCP}_{\mathrm{AR}}$ came from populations with the same distribution, we used the Wilcoxon Rank Sum test applying the continuity correction.

QUANTEC [16] reports that $V_{D}$, the volume receiving doses up to a specified threshold $D$, is not significantly associated with rectal toxicity for $\mathrm{D} \leq 45 \mathrm{~Gy}$ and provides dose-volume thresholds, with conventional fractionation up to $78 \mathrm{~Gy}$, which are more restrictive than our planning constraints: $\mathrm{V}_{50}<50 \%, \mathrm{~V}_{60}<35 \%, \mathrm{~V}_{65}<25 \%$, $\mathrm{V}_{70}<20 \%$ and $\mathrm{V}_{75}<15 \%$. Being widely accepted, the QUANTEC dose checkpoint values were used to describe the time behaviour of the relative volumes using the Gamma Distribution for the frequency of the relative rectum volumes at each QUANTEC dose value. 
Table 2. RTOG/EORTC toxicity score.

\begin{tabular}{|c|c|c|c|c|}
\hline $\begin{array}{c}\text { RTOG/EORTC } \\
\text { toxicity scale }\end{array}$ & Grade 1 & Grade 2 & Grade 3 & Grade 4 \\
\hline GI acute toxicity & $\begin{array}{l}\text { Increased frequency or } \\
\text { change in quality of } \\
\text { bowel habits not requiring } \\
\text { medication; } \\
\text { rectal discomfort not } \\
\text { requiring analgesics }\end{array}$ & $\begin{array}{l}\text { Diarrhoea requiring } \\
\text { parasympatholytic } \\
\text { drugs; mucous discharge not } \\
\text { necessitating sanitary pads; } \\
\text { rectal or abdominal pain } \\
\text { requiring analgesics }\end{array}$ & $\begin{array}{c}\text { Diarrhoea requiring } \\
\text { parenteral support; } \\
\text { severe mucous or blood } \\
\text { discharge } \\
\text { necessitating sanitary pads; } \\
\text { abdominal distension (flat plate } \\
\text { radiograph demonstrates } \\
\text { distended bowel loops) }\end{array}$ & $\begin{array}{c}\text { Obstruction. } \\
\text { fistula or perforation; GI } \\
\text { bleeding requiring } \\
\text { transfusion; abdominal } \\
\text { pain or } \\
\text { tenesmus requiring tube } \\
\text { decompression or bowel } \\
\text { diversion }\end{array}$ \\
\hline GI late toxicity & $\begin{array}{l}\text { Mild diarrhoea; mild } \\
\text { cramping; bowel } \\
\text { movements } 2-5 \\
\text { per day; slight } \\
\text { rectal discharge } \\
\text { or bleeding }\end{array}$ & $\begin{array}{c}\text { Moderate diarrhoea; } \\
\text { Intermittent severe cramping; } \\
\text { bowel movements > 5 per day; } \\
\text { moderate } \\
\text { excessive rectal } \\
\text { discharge; intermittent. } \\
\text { frequent bleeding } \\
\rightarrow \text { single laser } \\
\text { treatment and/ or } \\
\text { transfusion }\end{array}$ & $\begin{array}{l}\text { Watery diarrhoea; } \\
\text { obstruction } \\
\text { requiring surgery; } \\
\text { bleeding requiring } \\
\text { surgery or } 2 \\
\text { laser treatments } \\
\text { and/or transfusions }\end{array}$ & $\begin{array}{l}\text { Necrosis; perforation; } \\
\text { fistula; } \\
\text { abdominal pain or } \\
\text { tenesmus requiring tube } \\
\text { decompression } \\
\text { or bowel diversion }\end{array}$ \\
\hline
\end{tabular}

Therefore data of the percentage rectal volumes, have been collected from all DVHs of all the patients, both at the dose values corresponding to our constraints and at those of the QUANTEC constraints.

The Gamma Distribution was chosen, as data were positive and right-skewed. A wide distribution means high variability in the rectum volume receiving doses equal or higher than the chosen checkpoint value along the treatment course. On the contrary a narrow distribution means that the volume stays roughly the same. In the case of optimal treatment reproducibility, the curve becomes a Dirac's The Gamma Distribution parameters were estimated with a maximum likelihood estimator. For each patient and for each dose level (dose), the distributions were plotted superimposing the QUANTEC constraint $\left(V_{\text {dose }}^{Q}\right)$ and the percentage volume corresponding to the same dose level in $\mathrm{DVH}_{\mathrm{pCT}}\left(V_{\text {dose }}^{p C T}\right)$ and in $\mathrm{DVH}_{\mathrm{AR}}\left(V_{\text {dose }}^{A R}\right)$. These results were then employed to analyze how many patients had CBCT rectal volumes percentage outside the confidence interval established (95\%), i.e. the fraction of CBCT scans whose percentage rectal volumes, for each patient and QUANTEC dose-bin, statistically exceeded $V_{\text {dose }}^{Q}(1-p>0.05)$. The p value is, in fact, the integral of the gamma distribution up to $V_{\text {dose }}^{Q}$. This information has then been then qualitatively related to the follow up data.

As a further qualitative comparison, an empirical cumulative distribution function of the quantities $\mathrm{NTCP}_{\mathrm{pCT}}$, $\mathrm{NTCP}_{<\mathrm{DVH} 5>}, \mathrm{NTCP}_{<\mathrm{DVH}}$, $\mathrm{NTCP}_{\mathrm{AR} 5}$ and $\mathrm{NTCP}_{\mathrm{AR}}$ sampled on the 20 patients was calculated. Moreover we calculated the probability of having zero complications when the model adopted for the calculation of NTCPs of each patient was $\mathrm{NTCP}_{\mathrm{pCT}}$, $\mathrm{NTCP}_{<\mathrm{DVH} 5}, \mathrm{NTCP}_{<\mathrm{DVH}}>\mathrm{NTCP}_{\mathrm{AR} 5}$ and $\mathrm{NTCP}_{\mathrm{AR}}$, respectively. This was given by the product of the individual probabilities of having zero complications:

$$
\prod_{i=1}^{20}\left(1-N T C P_{i}\right)
$$

Statistical calculations were performed using the Mathematica8 (Wolfram Research, Inc. 100 Trade Center Drive, Champaign, IL 61820-7237, USA) and R version 2.15.1 (R Core Team, R Foundation for Statistical Computing, Vienna, Austria).

\section{Results}

Four patients (20\%) developed G2 acute gastrointestinal toxicity, no G3 acute or late toxicities were recorded. Only two patients (50\%) among those experiencing G2 acute toxicity presented co-morbidity and risk factors like diabetes, hypertension, and haemorroids (Figure 1). The Gamma Distributions of the rectum volumes at the QUANTEC dose levels were found to be highly variable among the patients depending on daily rectum filling. However, a trend towards smaller volumes as the treatment progressed was observed in most patients. As an 


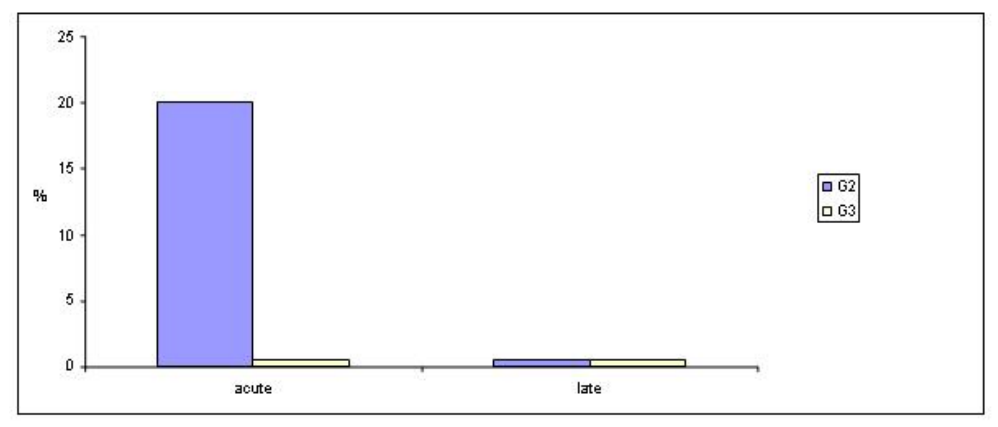

Figure 1. Gastrointestinal (GI) toxicity incidence.

example, in Figure 2 we show the Gamma Distribution plots relative to the percentage rectum volume receiving $65 \mathrm{~Gy}$ or more for two patients. The dashed vertical line represents the relative volume corresponding to the 65 Gy constraint specified by the QUANTEC $\left(V_{65}^{Q}\right)$, the dotted and continuous vertical lines are the relative volumes corresponding to $65 \mathrm{~Gy}$ in the DVH of the rectum in the pCT $\left(V_{65}^{p C T}\right)$ and in the AR $\left(V_{65}^{A R}\right)$, respectively. In the plot on the left (Figure 2(a)) a medium width distribution, corresponding to a fairly satisfactory repeatability of the rectum position is shown. Both $V_{65}^{p C T}$ and $V_{65}^{A R}$ are below $V_{65}^{Q}$ so the treatment plan would have been acceptable from the QUANTEC viewpoint and remained so during the treatment. In the plot on the right (Figure 2(b)) a slightly wider distribution is pictured, but in this case $V_{65}^{p C T}$ is higher than $V_{65}^{Q}$ while $V_{65}^{A R}$ is much lower. This is an example of a treatment plan that would fail acceptance criteria based on the QUANTEC hard constraints, which drifted towards an acceptable treatment thanks to the dieting regimen.

Inspecting all the distributions we have found that both $V_{50}^{p C T}$ and $V_{60}^{p C T}$ were lower than the QUANTEC constraints in 17 of the 20 patients (90\%); $V_{65}^{p C T}$ and $V_{70}^{p C T}$ were lower than $V_{65}^{Q}$ and $V_{70}^{Q}$ in 9 of the 20 patients (50\%) and in 15 of the 20 patients (75\%), respectively. $V_{75}^{p C T}$ was always below $V_{75}^{Q} \cdot V_{\text {dose }}^{A R}$ were always below the QUANTEC thresholds but in one patient, whose $V_{65}^{A R}$ was slightly above $V_{65}^{Q}$.

From the CBCT scans, our results indicate that, considering the p-value, 15 patients (75\%) had the percentage rectal volumes within $V_{50}^{Q}$; 14 (70\%), 11 (55\%), 13 (65\%) and 20 (100\%) patients had the percentage rectal volumes within $V_{60}^{Q}, V_{65}^{Q}, V_{70}^{Q}$ and $V_{75}^{Q}$, respectively.

The percentage of $\mathrm{DVH}_{\mathrm{pCT}}$ rectal volumes is almost always higher than the corresponding $\left\langle\mathrm{DVH}_{5}\right\rangle,\langle\mathrm{DVH}\rangle$, $\mathrm{DVH}_{\mathrm{AR} 5}$ and $\mathrm{DVH}_{\mathrm{AR}}$ suggesting that the dietary regimen was adequately followed during the treatment course. Small differences (with the exception of one patient) are evident comparing $<\mathrm{DVH}_{5}>$ with $<\mathrm{DVH}>$ and $\mathrm{DVH}_{\mathrm{AR} 5}$ and $\mathrm{DVH}_{\mathrm{AR}}$ (data not shown).

In Table 3 the Pearson product-moment correlation coefficients among all NTCPs, are displayed. All the coefficients relating $\mathrm{NTCP}_{\mathrm{pCT}}$ to the other indices are $<0.5$, indicating a weak correlation between the $\mathrm{DVH}$ of the pCT and the other DVHs. On the other hand, the coefficient between $\mathrm{NTCP}_{<\mathrm{DVH} 5>}$ and $\mathrm{NTCP}_{<\mathrm{DVH}>}$ as well between $\mathrm{NTCP}_{\mathrm{AR} 5}$ and $\mathrm{NTCP}_{\mathrm{AR}}$ were 0.883 and 0.865 , respectively, showing a substantial correlation.

In Table 4, the results of the Wilcoxon Rank Sum test among all NTCPs, are shown. We found that the null hypothesis (zero difference between the medians) could not be rejected ( $\mathrm{p}$-value $>0.05$ ) only for the couples $\left(\mathrm{NTCP}_{\mathrm{AR}}, \mathrm{NTCP}_{\mathrm{AR}}\right)$ and $\left(\mathrm{NTCP}_{<\mathrm{DVH} 5}\right.$, $\left.\mathrm{NTCP}_{<\mathrm{DVH}>}\right)$. For all the other couples of samples, with the possible exception of the couple ( $\left.\mathrm{NTCP}_{\mathrm{pCT}}, \mathrm{NTCP}_{<\mathrm{DVH}}\right)$, the p-values indicate that the difference between the corresponding medians is statistically significant. This finding agrees with the previous partial results.

Figure 3 shows the estimated cumulative distribution of the NTCP-QLKB values calculated from the $\mathrm{DVH}_{\mathrm{pCT}},<\mathrm{DVH} 5>,<\mathrm{DVH}>, \mathrm{DVH}_{\mathrm{AR}}$ and $\mathrm{DVH}_{\mathrm{AR}}$ in our patient population. NTCP data are binned at 0.001 intervals. $\mathrm{NTCP}_{\mathrm{AR}}$ and $\mathrm{NTCP}_{\mathrm{AR} 5}$ display a shift towards left corresponding to smaller values. This indicates that using these DVHs in calculating NTCP, a lower incidence of Grade $\geq 2$ late rectal toxicity is predicted with respect to the NTCPs calculated from the other DVHs. In addition, the values obtained on the 20 patients (maximum $\mathrm{NTCP}_{\mathrm{AR}} \sim 11 \%$ ) are smaller than those predicted from QUANTEC (Grade $\geq 2$ late rectal toxicity inferior 


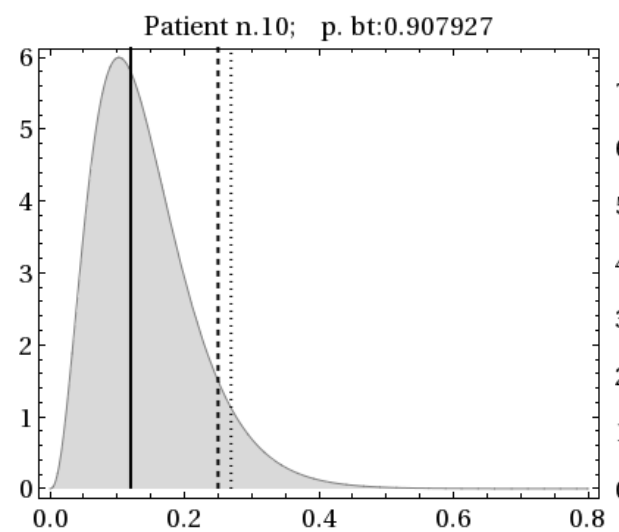

(a)

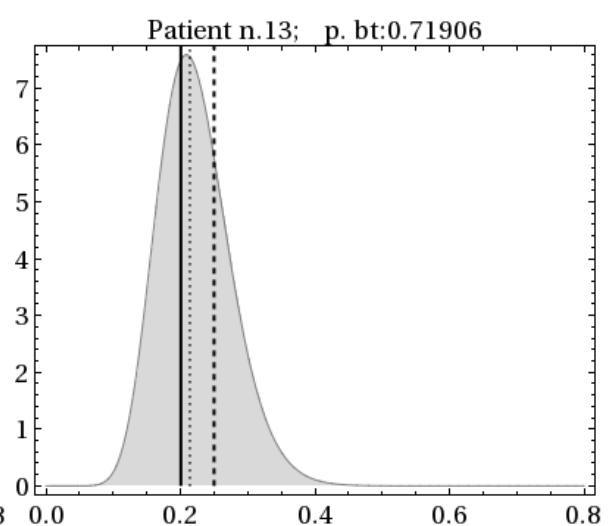

(b)

Figure 2. An example of gamma distributions plots for two patients and for the reference dose level of $65 \mathrm{~Gy}$; the red line is the value that corresponds to the percentage of volume established by QUANTEC constraints $\left(V_{65}^{Q}\right)$; the green line and the blue line are the value corresponding to the percentage of volume of AR and pCT respectively. The two plots correspond to two patients that both have $V_{65}^{p C T}$ higher than $V_{65}^{Q}$, but on the right $V_{65}^{A R}$ is higher than $V_{65}^{Q}$, as well, while on the left it is less than $V_{65}^{Q}$ indicating an improvement of the DVH during the treatment.

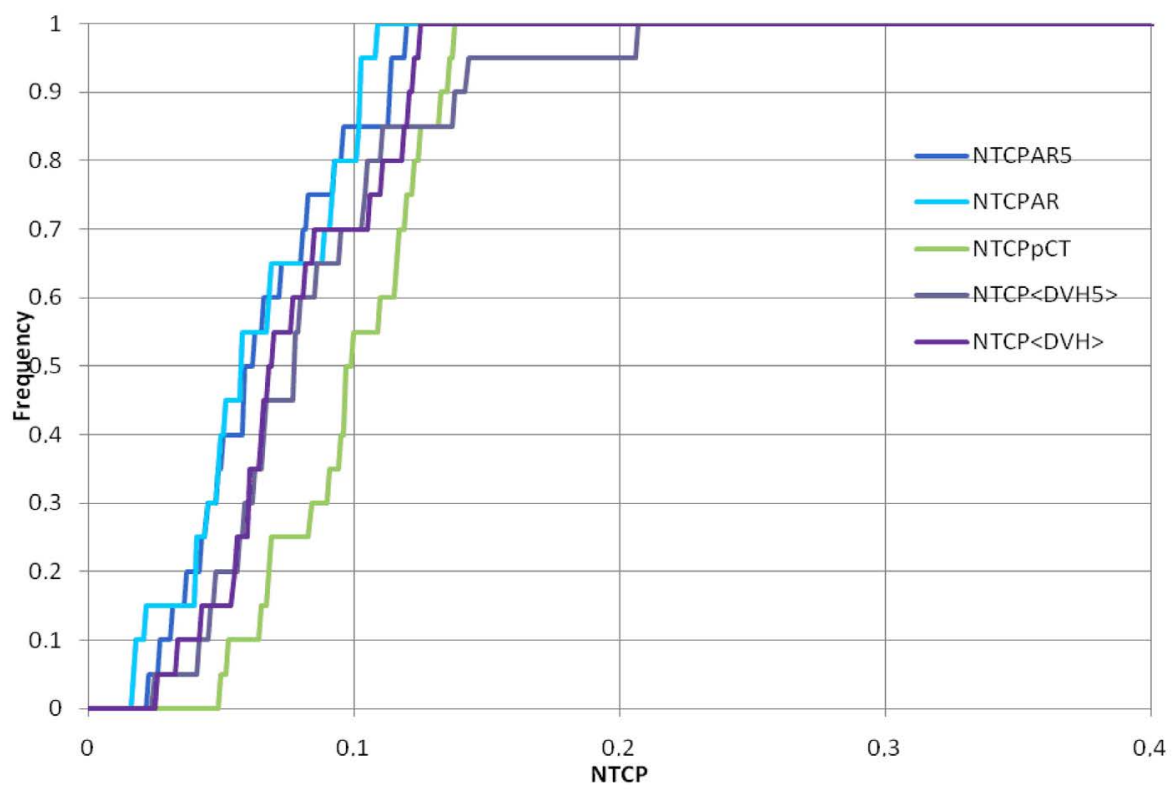

Figure 3. Estimated cumulative distributions of NTCP-QLKB values calculated from the DVHs of pCT, $<$ DVH5 $>$, $<$ DVH $>$, AR5 and AR in the entire patient population.

to $15 \%)$ and of about the same order for the probability of Grade $\geq 3(10 \%)$. This is consistent with the fact that the percentage volumes obtained for AR are smaller than the thresholds accepted in the QUANTEC review, as well.

The values of the probability of having zero complications ranged range from a minimum of $12 \%$ for $\mathrm{NTCP}_{\mathrm{pCT}}$ to maximum of $26 \%$ for $\mathrm{NTPC}_{\mathrm{AR}}$.

\section{Discussion}

In the last years, different studies have shown that dose escalation to the prostate can improve clinical outcomes 
Table 3. Pearson's correlation coefficients among the NTCP values calculated on the corresponding DVHs.

\begin{tabular}{cccccc}
\hline & \multicolumn{5}{c}{ Pearson's correlation coefficient } \\
\hline & $\mathrm{NTCP}_{\mathrm{PCT}}$ & $\mathrm{NTCP}_{<\mathrm{DVH5}>}$ & $\mathrm{NTCP}_{<\mathrm{DVH}>}$ & $\mathrm{NTCP}_{\mathrm{AR} 5}$ & $\mathrm{NTCP}_{\mathrm{AR}}$ \\
\hline $\mathrm{NTCP}_{\mathbf{p C T}}$ & 1 & 0.390 & 0.424 & 0.387 & 0.369 \\
$\mathrm{NTCP}<$ DVH5 $>$ & 0.390 & 1 & 0.883 & 0.894 & 0.678 \\
$\mathrm{NTCP}<$ DVH> & 0.424 & 0.883 & 1 & 0.900 & 0.894 \\
$\mathrm{NTCP}_{\text {AR5 }}$ & 0.387 & 0.894 & 0.900 & 1 & 0.865 \\
$\mathrm{NTCP}_{\text {AR }}$ & 0.369 & 0.678 & 0.894 & 0.865 & 1 \\
\hline
\end{tabular}

Table 4. Wilcoxon Rank Sum test, comparing two related samples in order to assess whether their population mean ranks differ.

\begin{tabular}{|c|c|c|c|c|}
\hline \multicolumn{3}{|c|}{$\begin{array}{l}\text { 95\% Confidence intervals of the Wilcoxon } \\
\text { rank sum test }\end{array}$} & \multirow[b]{2}{*}{$\mathrm{NTCP}_{\text {AR5 }}$} & \multirow[b]{2}{*}{$\mathrm{NTCP}_{\mathrm{AR}}$} \\
\hline & $\mathrm{NTCP}_{<\mathrm{DVH} 5>}$ & $\mathrm{NTCP}_{<\mathrm{DVH}>}$ & & \\
\hline $\mathrm{NTCP}_{\mathbf{p C T}}$ & $\begin{array}{l}-0.002-0.033 \\
(p-\text {-value }=0.08)\end{array}$ & $\begin{array}{c}0.005-0.037 \\
(\mathrm{p} \text {-value }<0.01)\end{array}$ & $\begin{array}{c}0.002-0.05 \\
\text { (p-value }<0.01)\end{array}$ & $\begin{array}{c}0.020-0.051 \\
(\mathrm{p}-\text {-value }<0.01)\end{array}$ \\
\hline $\mathrm{NTCP}_{<\text {DVH5 }>}$ & & $\begin{array}{l}-0.003-0.015 \\
(p-\text { value }=0.19)\end{array}$ & $\begin{array}{c}0.009-0.020 \\
(p-\text { value }<0.01)\end{array}$ & $\begin{array}{c}0.008-0.032 \\
(p-\text { value }<0.01)\end{array}$ \\
\hline $\mathrm{NTCP}_{<\mathbf{D V H}>}$ & & & $\begin{array}{c}0.005-0.020 \\
(p-\text { value }<0.01)\end{array}$ & $\begin{array}{c}0.011-0.018 \\
(\mathrm{p}-\text {-value }<0.01)\end{array}$ \\
\hline $\mathrm{NTCP}_{\text {AR5 }}$ & & & & $\begin{array}{c}-0.005-0.011 \\
(p-\text { value }=0.38)\end{array}$ \\
\hline
\end{tabular}

[3] [4] but may increase the risk of rectal toxicity [29]. A number of consistent results on dose-volume effect and rectal bleeding appeared in literature and most of them confirmed the rectum behaving prevalently as a serial organ. In particular, significant correlations between late rectal bleeding and the fraction of rectal volume receiving more than 40 - $60 \mathrm{~Gy}\left(\mathrm{~V}_{40}-\mathrm{V}_{60}\right.$ : intermediate dose) and more than 65 - $75 \mathrm{~Gy}\left(\mathrm{~V}_{65}-\mathrm{V}_{75}\right.$ : high dose) have been reported [10]-[17] [30]-[34].

The QUANTEC group published a review analyzing dose-volume data from multiple centers using the LKB NTCP model. Dose-volume constraints were established for conventional fractionation up to 79.2 Gy, considered as conservative and starting from 3D treatment planning. Following these constraints, the probability of Grade $\geq 2$ and Grade $\geq 3$ late rectal toxicity is expected to decrease to $<15 \%$ and to $<10 \%$. In the above mentioned papers patients were asked to keep their rectum empty during therapy, but the specific role of a diet on the radiobiological parameters has not been extensively investigated yet, even though the benefit of a dietary protocol in combination with an IGRT protocol is well assessed in literature [25] and confirmed by our previous results [26].

In our previous investigation, we proposed a method to evaluate the dose delivered to the rectum during the course of treatment using the information of an off-line definition of organ volume using a commercial linear accelerator with an integrated CBCT scanner [26]. We verified on 6 patients that, taking into account only the volumes corresponding to 4 dose points $\left(\mathrm{V}_{40}, \mathrm{~V}_{50}, \mathrm{~V}_{60}\right.$ and $\left.\mathrm{V}_{70}\right)$, that both $\mathrm{DVH}_{\mathrm{AR}}$ and $<\mathrm{DVH}>$ could describe the average behaviour of the rectum during the treatment course. We also observed that $\mathrm{DVH}_{\mathrm{pCT}} \mathrm{appeared}$ higher than the other DVHs in all six patients and we interpreted it as a proof of the need to stress the diet and bowel cleaning, probably not yet optimal at the beginning of the treatment. It remained to be cleared which of the two DVHs should be used to best predict rectum toxicities and whether the CBCT scans performed during the first week of treatment could be taken as representative of the whole treatment. In this paper, the aim was to confirm the importance of a “diet-IGRT” combination and to try to find an answer to the above questions.

Following the patients during the whole treatment, with the help of the CBCT scans, we were able to check the rectum filling and to assess if patients really followed the diet. All DVH values improve as confirmed by follow-up data which show that $20 \%$ of patients present G2 acute toxicity while acute G3 toxicity and late toxicity have been not recorded. Indeed, starting from late toxicity data (zero), our results show that, in the planning CT, QUANTEC constraints were not satisfied in some patients. During the treatment, however, this number decreased thanks to the diet, and the number of CBCT scans respecting dose-volume constraints increased, as 
shown by the Gamma Distributions. The QUANTEC constraints are more restrictive than those applied in our institute and the fact that in the case of $\mathrm{DVH}_{\mathrm{AR}}$ they are always satisfied is consistent with our follow-up data showing no late rectal toxicity. The analysis of Gamma Distributions can help us to have off-line information on the reproducibility of the treatment, as well as on the reproducibility of the dietary regimen followed by each patient and, therefore, on the strength of the entire procedure. From Gamma distributions, in addition, we found that the number of patients with a $V_{\text {dose }}^{p C T}$ lower than $V_{\text {dose }}^{Q} \quad$ increases with dose and all $V_{75}^{p C T}$ values are lower than $V_{75}^{Q}$ ones. This finding well agrees with our toxicity data (zero) and with literature which reports that late rectal toxicity is mostly related to the size of the high dose region [10]-[17] [30]-[34].

The number of patient analyzed is too small to correlate the data for acute toxicity with clinical factors. However, some general considerations can be extracted from Gamma Distributions. The four patients who showed acute toxicity are those with the widest Gamma Distributions, i.e. patients whose treatment was least reproduceble. In addition, three of the four have all $V_{\text {dose }}^{p C T}$ values, but $V_{75}^{p C T}$ higher that $V_{\text {dose }}^{Q}$ and at least $30 \%$ of the Gamma Distribution above the QUANTEC constraints. In literature, a correlation between acute and late rectal toxicity has been observed although its nature is not well established [29] [35] [36]. In our small population, the four patients who experienced acute toxicity, did not show late toxicity, and this suggests that among all dose-volume constraints, the $\mathrm{V}_{75}$, which was always respected in the pCT, in all the CBCT scans and in AR, turned out to be the most consistent with the hypothetical correlation between acute and late toxicity.

To further investigate the open questions about the model that best summarizes the rectum movements along the treatment course, we performed a statistical analysis in terms of NTCP. We decided to use the NTCP-LKB model with QUANTEC best estimate parameters because of the low number of patients that doesn't allow us to make clinical distinctions among them.

The Pearson's correlation coefficients indicate a substantial correlation between all indices, except for $\mathrm{NTCP}_{\mathrm{pCT}}$, with a general positive trend. The Wilcoxon Rank Sum test, however, indicates that the difference between the medians for the couple $\left(\mathrm{NTCP}_{\mathrm{AR}}, \mathrm{NTCP}_{\mathrm{AR}}\right)$ and for the couple $\left(\mathrm{NTCP}_{<\mathrm{DVH} 5}\right.$, $\left.\mathrm{NTCP}_{<\mathrm{DVH}>}\right)$ is not statistically significant (p-value $>0.05$ ). For the other couples of samples the p-values indicate that the differences are statistically significant (p-value $<0.01$ ), i.e. it is not ruled out the possibility that the rectum behavior during the whole treatment course can be represented by the first treatment week.

To further investigate this, we have then compared the NTCP values with the results of the follw-up. From NTCP distributions (Figure 3), the best predictors for zero late rectal toxicity seem to be AR and AR5 (lower values and narrower distributions). In addition, the highest probability to have no complications on 20 patients is obtained with $\mathrm{NTCP}_{\mathrm{AR}}(26 \%)$. These findings suggest that the AR model seems the more consistent with patients' outcome and that, when the treatment is reproducible along the entire treatment course, which can be verified by CBCT scans, the $\mathrm{DVH}_{\mathrm{AR} 5}$ well describes the rectum behavior.

\section{Conclusions}

At the end of our analysis, we conclude that if we concentrate on AR for all patients our results are in a good agreement with QUANTEC assertions and follow-up data. In fact, we obtain that $\mathrm{DVH}_{\mathrm{AR}}$ always respects QUANTEC constraints and we record no late toxicity in our follow-up data. In addition, when the rectum filling remains reproducible during the entire course of treatment, the $\mathrm{DVH}_{\mathrm{AR} 5}$ can describe the rectum behavior in the whole treatment. Finally, from the Gamma distributions, and from our toxicity data, we can suggest the $\mathrm{V}_{75}$ as the best predictor of late rectal toxicity. In any case, we are enrolling more patients and performing more statistical analysis to confirm these preliminary results.

Although our analysis has the limit of being extended to very few patients, these preliminary results show that a dietary protocol associated with IGRT technique in order to daily verify the correct dieting behaviour is useful to avoid toxicities on the anterior wall of the rectum.

\section{Conflict of Interest}

The authors declare that they have no conflict of interest.

\section{References}

[1] Jemal, A., Siegel, R., Ward, E., Hao, Y., Xu, J. and Thun, M.J. (2009) Cancer Statistics, 2009. CA: A Cancer Journal 
for Clinicians, 59, 225-249. http://dx.doi.org/10.3322/caac.20006

[2] Ferlay, J., Autier, P., Boniol, M., Heanue, M., Colombet, M. and Boyle, P. (2007) Estimates of Cancer Incidence and mortality in Europe in 2006. Annals of Oncology, 18, 581-592. http://dx.doi.org/10.1093/annonc/mdl498

[3] Peschel, R.E. and Colberg, J.W. (2003) Surgery, Brachytherapy, and External-Beam Radiotherapy for Early Prostate Cancer. Lancet Oncology, 4, 233-241. http://dx.doi.org/10.1016/S1470-2045(03)01035-0

[4] Kuban, D.A., Tucker, S.L., Dong, L., Starkschall, G., Huang, E.H., Cheung, M.R., Lee, A.K. and Pollack, A. (2008) Long-Term Results of the M. D. Anderson Randomized Dose-Escalation Trial for Prostate Cancer. International Journal of Radiation Oncology*Biology*Physics, 70, 67-74. http://dx.doi.org/10.1016/j.ijrobp.2007.06.054

[5] Kupelian, P.A., Reddy, C.A., Carlson, T.P., Altsman, K.A. and Willoughby, T.R. (2002) Preliminary observations on biochemical relapse-free survival rates after short-course intensity-modulated radiotherapy (70 Gy at 2.5 Gy/fraction) for localized prostate cancer. International Journal of Radiation Oncology*Biology*Physics, 53, 904-912. http://dx.doi.org/10.1016/S0360-3016(02)02836-5

[6] Herold, D.M., Hanlon, A.L. and Hanks, G.E. (1999) Diabetes Mellitus: A Predictor for Late Radiation Morbidity. International Journal of Radiation Oncology*Biology*Physics, 43, 475-479. http://dx.doi.org/10.1016/S0360-3016(98)00460-X

[7] Peeters, S.T., Lebesque, J.V., Heemsbergen, W.D., van Putten, W.L., Slot, A., Dielwart, M.F., et al. (2006) Localized Volume Effects for Late Rectal and Anal Toxicity after Radiotherapy for Prostate Cancer. International Journal of Radiation Oncology*Biology*Physics, 64, 1151-1161. http://dx.doi.org/10.1016/j.ijrobp.2005.10.002

[8] Vavassori, V., Fiorino, C., Rancati, T., Magli, A., Fellin, G., Baccolini, M., et al. (2007) Predictors for Rectal and Intestinal Acute Toxicities during Prostate Cancer High-Dose 3D-CRT: Results of a Prospective Multicenter Study. International Journal of Radiation Oncology*Biology*Physics, 67, 1401-1410. http://dx.doi.org/10.1016/j.ijrobp.2006.10.040

[9] Liu, M., Pickles, T., Agranovich, A., Berthelet, E., Duncan, G., Keyes, M., et al. (2004) Impact of Neoadjuvant Androgen Ablation and Other Factors on Late Toxicity after External Beam Prostate Radiotherapy. International Journal of Radiation Oncology*Biology*Physics, 58, 59-67.

[10] Boersma, L.J., van den Brink, M., Bruce, A.M., Shouman, T., Gras, L., Annet te Velde, et al. (1998) Estimation of the Incidence of Late Bladder and Rectum Complications after High-Dose (70-78 Gy) Conformal Radiotherapy for Prostate Cancer, Using Dose-Volume Histograms. International Journal of Radiation Oncology*Biology*Physics, 41, 8392.

[11] Fiorino, C., Sanguineti, G., Cozzarini, C., Fellin, G., Foppiano, F., Menegotti, L., et al. (2003) Rectal Dose-Volume Constraints in High-Dose Conformal Radiotherapy for Localized Prostate Cancer. International Journal of Radiation Oncology*Biology*Physics, 57, 953-962.

[12] Rancati, T., Fiorino, C., Gagliardi, G., Cattaneo, G.M., Sanguineti, G., Borca, V.C., et al. (2004) Fitting Late Rectal Bleeding Data Using Different NTCP Models: Results from an Italian Multi-Centric Study (AIROPROS0101). Radiotherapy and Oncology, 73, 21-32. http://dx.doi.org/10.1016/j.radonc.2004.08.013

[13] Fellin, G., Fiorino, C., Rancati, T., Valvassori, V., Barra, S., Cagna, E., et al. (2008) Late Rectal Bleeding after Conformal Radiotherapy for Prostate Cancer: NTCP Modelling. Radiotherapy and Oncology, 88, S332-S333.

[14] Fiorino, C., Fellin, G. and Rancati, T. (2008) Clinical and Dosimetric Predictors of Late Rectal Syndrome after 3DCRT for Localized Prostate Cancer: Preliminary Results of a Multicenter Prospective Study. International Journal of Radiation Oncology*Biology*Physics, 70, 1130-1137.

[15] Gulliford, S.L., Foo, K., Morgan, R.C., Aird, E.G., Bidmead, A.M., Critchley, H., et al. (2010) Volume Constraints to Reduce Rectal Side Effects from Prostate Radiotherapy: Evidence from mrc rt01 Trial isrctn 47772397. International Journal of Radiation Oncology*Biology*Physics, 76, 747-754.

[16] Michalski, J.M., Gay, H., Jackson, A., Tucker, S.L. and Deasy, J.O. (2010) Radiation Dose-Volume Effects in Radiation-Induced Rectal Injury. International Journal of Radiation Oncology Biology Physics, 76, S123-S129.

[17] Rancati, T., Fiorino, C., Fellin, G., Vavassori, V., Cagna, E., Borca, V.C., et al. (2011) Inclusion of Clinical Risk Factors into NTCP Modelling of Late Rectal Toxicity after High Dose Radiotherapy for Prostate Cancer. Radiotherapy and Oncology, 100, 124-130. http://dx.doi.org/10.1016/j.radonc.2011.06.032

[18] Onal, C., Topkan, E., Efe, E., Yavuz, M., Sonmez, S. and Yavu, A. (2009) Comparison of Rectal Volume Definition Techniques and Their Influence on Rectal Toxicity in Patients with Prostate Cancer Treated with 3D Conformal Radiotherapy: A Dose-Volume Analysis. Radiation Oncology, 4, 14.

[19] Boehmer, D., Kuczer, D., Badakhshi, H., Stiefel, S., Kuschke, W., Wernecke, K.D. and Budach, V. (2006) Influence of Organ at Risk Definition on Rectal Dose-Volume Histograms in Patients with Prostate Cancer Undergoing ExternalBeam Radiotherapy. Strahlentherapie und Onkologie, 82, 277-282. http://dx.doi.org/10.1007/s00066-006-1462-7

[20] O’Donnel, H.E., Finnegan, K., Eliades, H., Oliveros, S. and Plawman, P.N. (2008) Re-Defining Rectal Volume and 
DVH for Analysis of Rectal Morbidity Risk after Radiotherapy for Early Prostate Cancer. The British Journal of Radiology, 81, 327-332. http://dx.doi.org/10.1259/bjr/75868623

[21] Kupelian, A.P., Langen, K.M., Willoughby, T.R., Zeidan, O.A. and Sanford, L.M. (2008) Image-Guided Radiotherapy for Localized Prostate Cancer: Treating a Moving Target. Seminars in Radiation Oncology, 18, 58-66. http://dx.doi.org/10.1016/j.semradonc.2007.09.008

[22] Huang, K., Palma, D.A., Scott, D., McGregor, D., Gaede, S., Yartsev, S., Bauman, G., Louie, A.V. and Rodrigues, G. (2012) Inter- and Intrafraction Uncertainty in Prostate Bed Image-Guided Radiotherapy. International Journal of Radiation Oncology*Biology*Physics, 84, 402-407. http://dx.doi.org/10.1016/j.ijrobp.2011.12.035

[23] Thor, M., Bentzen, L., Hysing, L.B., Ekanger, C., Helle, S.I., Karlsdóttir, Á., et al. (2013) Prediction of Rectum and Bladder Morbidity Following Radiotherapy of Prostate Cancer Based on Motion-Inclusive Dose Distributions. Radiotherapy and Oncology, 107, 147-152. http://dx.doi.org/10.1016/j.radonc.2013.03.029

[24] Thor, M., Væth, M., Karlsdottir, Á. and Muren, L.P. (2010) Rectum Motion and Morbidity Prediction: Improving Correlation between Late Morbidity and DVH Parameters through Use of Rectum Planning Organ at Risk Volumes. Acta Oncologica, 49, 1061-1068. http://dx.doi.org/10.3109/0284186X.2010.505200

[25] Smitsmans, M.H.P., Floris, J.P., de Bois, R.T.T.J., Wilma, D., Heemsbergen, W.D., Sonke, J.J., Lebesque, J.V., et al. (2008) The Influence of a Dietary Protocol on Cone Beam CT-Guided Radiotherapy for Prostate Cancer Patients. International Journal of Radiation Oncology*Biology*Physics, 71, 1279-1286.

[26] Falco, M.D., D’Andrea, M., Fedele, D., Barbarino, R., Benassi, M., Giudice, E., et al. (2011) Preliminary Experience of a Predictive Model to Define Rectal Volume and Rectal Dose during the Treatment of Prostate Cancer. The British Journal of Radiology, 84, 819-825. http://dx.doi.org/10.1259/bjr/25741415

[27] Kutcher, G.J., Burman, C., Brewster, L., Goitein, M. and Mohan, R. (1991) Histogram Reduction Method for Calculating Complication Probabilities for Three Dimensional Treatment Planning Evaluations. International Journal of Radiation Oncology*Biology*Physics, 21, 137-146.

[28] Falco, M.D., Fontanarosa, D., Miceli, R., Carosi, A., Santoni, R. and D’Andrea, M. (2011) Preliminary Studies for a CBCT Imaging Protocol for Off-Line Organ Motion Analysis: Registration Software Validation and CTDI Measurements. Medical Dosimetry, 36, 91-101. http://dx.doi.org/10.1016/j.meddos.2010.01.003

[29] Peeters, S.T., Heemsbergen, W.D., van Putten, W.L.J., Scot, A., Tabak, H., Mens, J.W., et al. (2005) Acute and Late Complications after Radiotherapy for Prostate Cancer: Results of a Multicenter Randomized Trial Comparing 68 Gy to 78 Gy. International Journal of Radiation Oncology*Biology*Physics, 61, 1019-1034.

[30] Liu, M., Berthelet, E., Patterson, K., Dick, K. and Kwan, W. (2003) Various Techniques of Contouring the Rectum and Their Impact on Rectal Dose-Volume Histograms. Medical Dosimetry, 28, 189-192. http://dx.doi.org/10.1016/S0958-3947(03)00071-2

[31] Peeters, S.T.H., Hoogeman, M.S., Heemsbergen, W.D., Hart, A.A.M., Koper, P.C.M. and Lebesque, J.V. (2006) Rectal Bleeding, Fecal Incontinence and High Stool Frequency after Conformal Radiotherapy for Prostate Cancer: Normal Tissue Complication Probability Modelling. International Journal of Radiation Oncology Biology Physics, 66, 11-19.

[32] Huang, E.H., Pollack, A., Levy, L., Starkschall, G., Dong, L., Rosen, I., et al. (2002) Late Rectal Toxicity: Dose-Volume Effects of Conformal Radiotherapy for Prostate Cancer. International Journal of Radiation Oncology*Biology* Physics, 54, 1009-1119.

[33] Nuyttens, J.J., Milito, S., Rust, P.F. and Turrisi, A.T. (2002) Dose-Volume Relationship for Acute Side Effects during High Dose Conformal Radiotherapy for Prostate Cancer. Radiotherapy and Oncology, 64, 209-214. http://dx.doi.org/10.1016/S0167-8140(02)00185-8

[34] Marzi, S., Arcangeli, G., Saracino, B., Petrongari, M.G., Bruzzaniti, V., Iaccarino, G., et al. (2007) Relationships between Rectal Wall Dose-Volume Constraints and Radiobiologic Indices of Toxicity for Patients with Prostate Cancer. International Journal of Radiation Oncology*Biology*Physics, 68, 41-49.

[35] Vargas, C., Martinez, A., Kestin, L.L., Yan, D., Grills, I., Brabbins, D.S., et al. (2005) Dose Volume Analysis of Predictors for Chronic Rectal Toxicity after Treatment of Prostate Cancer with Adaptive Image-Guided Radiotherapy. International Journal of Radiation Oncology*Biology*Physics, 62, 1297-1308.

[36] Zelefsky, M.J., Levin, E.J., Hunt, M., Yamada, Y., Shippy, A.M., Jackson, A. and Amols, H.I. (2008) Incidence of Late Rectal and Urinary Toxicities after Three-Dimensional Conformal Radiotherapy and Intensity-Modulated Radiotherapy for Localized Prostate Cancer. International Journal of Radiation Oncology*Biology*Physics, 70, 1124-1129. 
Scientific Research Publishing (SCIRP) is one of the largest Open Access journal publishers. It is currently publishing more than 200 open access, online, peer-reviewed journals covering a wide range of academic disciplines. SCIRP serves the worldwide academic communities and contributes to the progress and application of science with its publication.

Other selected journals from SCIRP are listed as below. Submit your manuscript to us via either submit@scirp.org or Online Submission Portal.
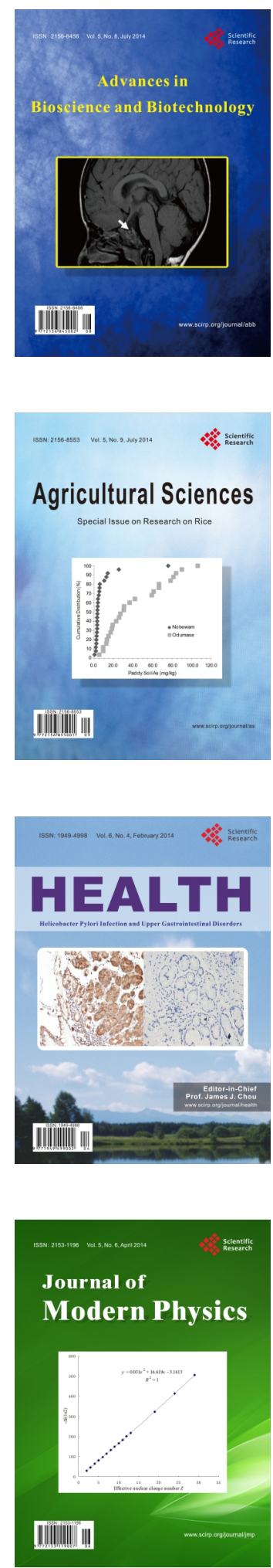
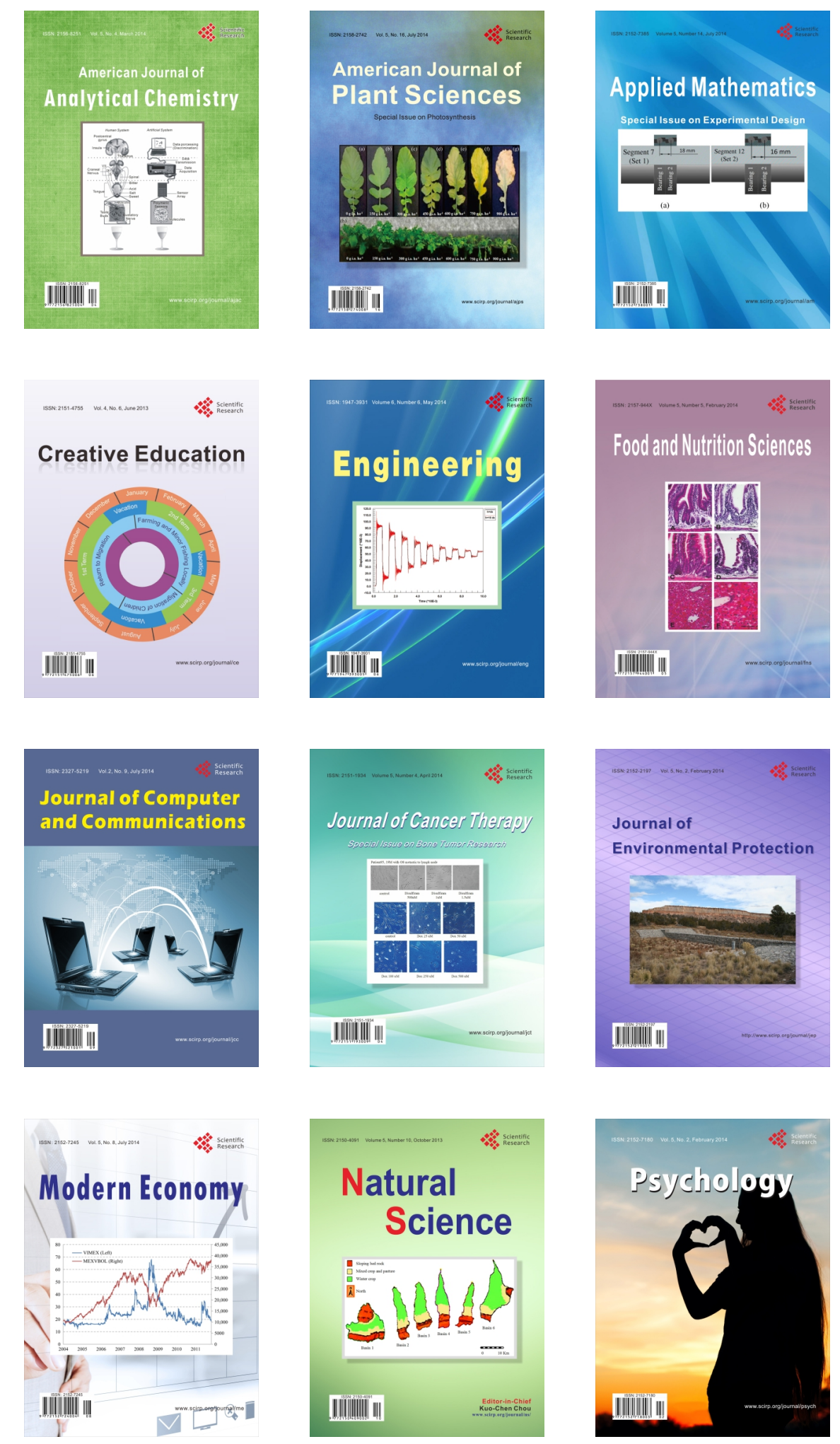